\title{
Effect of stimulus repetition on positive and negative identity priming
}

\author{
GREGORY B. MALLEY and DAVID L. STRAYER \\ University of Utah, Salt Lake City, Utah
}

\begin{abstract}
Most negative-priming experiments have used a limited number of stimuli that are repeated many times throughout the experiment. We report five experiments that examine in greater detail the role of stimulus repetition in negative priming. Subjects were presented with displays consisting of two or more words, and were required to name the word printed in red. On attended repetition (AR) trials, the target word was the same as the target word on the preceding trial. On ignored repetition (IR) trials, the target word was the same as the distractor word on the preceding trial. Experiments 1 and 2 used novel words, and obtained positive priming on AR trials, but no negative priming on IR trials. Experiments 3 and 4 used repeated words, and obtained negative priming on IR trials, but no positive priming on AR trials. In Experiment 5, both novel and repeated words were intermixed, and negative priming was observed for repeated, but not novel, IR conditions, whereas positive priming was observed for novel, but not repeated, AR conditions. Together, Experiments 1-5 demonstrate that positive and negative identity priming are modulated by stimulus repetition and are stimulus specific.
\end{abstract}

How targets are successfully selected among distractors in the stimulus environment is an important issue within cognitive psychology. Many theories (e.g., Broadbent, 1958; Treisman, 1964; Van der Heijden, 1981) have explained selective attention in terms of increased processing and activation levels for relevant target items. The key idea behind these theories is that relevant items receive more activation than irrelevant items. However, since the seminal studies of Neill $(1977,1979)$ and Tipper (1985), a great deal of research has focused on the fate of irrelevant information, and has caused a reevaluation of these theories.

Tipper (1985) presented subjects with two superimposed objects and required them to name the object presented in green and ignore the object presented in red. Positive identity and semantic priming effects emerged, the former when the target objects in the prime and probe trials were identical, and the latter when they were semantically related. In contrast, reaction time (RT) was significantly lengthened when a distractor on a prime trial was subsequently presented as a probe target on the succeeding trial. This effect occurred in both identity and semantic conditions. Tipper introduced the term negative priming, and suggested that targets and distractors are processed differently by the information-processing system. Positive priming reflects the activation of an item above a base level of activation, whereas negative prim-

We are indebted to Melissa Heckenlaible, Shelly Dockstader, and Trudi Walkley for assistance in data collection. We would like to thank William Johnston, Lester Krueger, Robert Proctor, and two anonymous reviewers for comments on a previous version of this article. Correspondence concerning this article should be addressed to D. L. Strayer, Department of Psychology, University of Utah, Salt Lake City, UT 84112 (e-mail: strayer@freud.sbs.utah). ing reflects the inhibition of an item below a base level of activation.

The negative-priming effect is robust. It has been observed in a variety of tasks, including naming (Tipper, 1985; Tipper \& Cranston, 1985), lexical decision (Neumann, McCloskey, \& Felio, 1993; Yee, 1991), matching (DeSchepper \& Treisman, 1991; Neill, Lissner, \& Beck, 1990; Treisman, 1992), categorization (Tipper \& Baylis, 1987; Tipper \& Driver, 1988), and localization (Tipper, Brehaut, \& Driver, 1990). The negative-priming effect has also been obtained with a variety of stimuli, including objects (Tipper, 1985), letters (Neumann \& DeSchepper, 1992; Tipper \& Cranston, 1985), words (Hasher, Kane, Stoltzfus, Zacks, \& Connelly, in press; Neill, 1977), and nonsense shapes (DeSchepper \& Treisman, 1991; Treisman, 1992).

The negative-priming effect has also been demonstrated both when vocal responses (Neill, 1977; Tipper, 1985; Tipper \& Cranston, 1985) and when manual responses (Neumann and DeSchepper, 1992; Tipper et al., 1990) have been used. More importantly, the effect can be obtained when response to the prime is in one modality and response to the probe is in another modality (Tipper, MacQueen, \& Brehaut, 1988). This result suggests that the negative-priming mechanism occurs at a relatively central processing location, and is not associated with within-modality interference.

What do these empirical results tell us about selective attention? They suggest that successful selective attention is a combination of target activation and distractor inhibition. For a human information processor to be efficient at selection, mechanisms of activation and inhibition need to be present. Not all humans seem to possess such a mechanism of inhibition. For example, schizophrenics show no negative-priming effects (Beech, Baylis, 
Smithson, \& Claridge, 1989; Beech \& Claridge, 1987). This suggests that schizophrenics are unable to inhibit distracting information, and that "normal" efficient information processing is associated with a functioning inhibitory mechanism.

Negative-priming experiments have typically used a limited set of stimuli that are repeated throughout the experiment. Thus, it is quite possible that negative priming depends on stimulus repetition. The repetition may serve to highly activate the mental representations of those stimuli. Under these circumstances, the information-processing system must determine which of two highly activated items is the target item. As the activation levels of targets and distractors increase, selection difficulty should increase, thereby increasing the negative-priming effect. An important exception to the generalization that negative-priming effects have been obtained with a limited stimulus set comes from the studies recently reported by DeSchepper and Treisman (1991; see also Treisman, 1992). DeSchepper and Treisman reported that negative priming for novel shapes persisted for up to 200 intervening trials. They concluded that a single presentation of a novel shape is sufficient to establish an independent internal representation of that shape. One interpretation of these results is that negative priming does not depend on stimulus repetition; negative priming will emerge for novel, as well as for repeated, stimuli. However, this interpretation may be premature for several reasons.

First, DeSchepper and Treisman's (1991) "novel" shapes may not have been truly novel within their experiments. They themselves alluded to this possibility by noting that "inhibition appears to last at full strength and specificity across 200 intervening trials using very similar shapes" (p. 7, our emphasis). It is possible that the repetition of "very similar" shapes resulted in the development of a prototype representation of these shapes.

Research on categorization (e.g., Rosch, 1975) has suggested that mental categories are represented in terms of a prototype, and that prototypes have ill-defined and uncertain boundaries. The categorization literature suggests that it is unlikely that the subjects in DeSchepper and Treisman's (1991) studies created independent internal representations of each of the 260 nonsense shapes. Moreover, retaining these independent internal representations through 200 intervening trials imposes a considerable burden on memory. One possible explanation for the discrepancy between DeSchepper and Treisman's data and the results of previous research using repeated stimuli is that the 260 nonsense shapes may have been classified into a limited set of categories. Thus, the repetition of highly similar shapes in DeSchepper and Treisman's experiments may have been analogous to the familiar stimulus conditions used by other researchers.

Moreover, DeSchepper and Treisman's (1991) stimuli were not all novel. In each experiment, novel and familiar shapes were intermixed. For example, Experiment 1 started with a prefamiliarization stage consisting of 10 repeated shapes. Trials with novel shapes were then inter- mixed with those of familiar shapes. The familiar stimuli may have facilitated the obtained negative priming.

In summary, negative priming has been primarily obtained in experiments that employ a limited number of stimuli that are repeated many times throughout the experiment. One notable exception consists of the data reported by DeSchepper and Treisman (1991). However, it is unclear how subjects treated the novel stimuli in these experiments. Thus, the question as to whether negative priming will be obtained with completely novel stimuli (i.e., stimuli that are seen only twice in the experiment, once as a distractor, and once as a target) remains unclear.

We examined two types of priming conditions in each of the five experiments reported in this article. On attended repetition (AR) trials, the target on the probe trial was the same as the target on the prime trial. On ignored repetition (IR) trials, the target on the probe trial was the same as the distractor on the prime trial. The priming literature suggests that we should obtain negative priming on IR trials and positive priming on AR trials. Moreover, whereas the repetition-priming literature suggests that positive priming (measured as the difference in performance between prime and probe) should diminish with stimulus repetition (e.g., Logan, 1990), the negative-priming literature is mute with respect to the effect of stimulus repetition on negative identity priming (but see DeSchepper \& Treisman, 1991).

The primary purpose of the present research was to investigate the extent to which negative priming depends on stimulus repetition. We also examined two additional factors thought to influence negative priming. First, the effect of an intervening trial between the prime and probe stimuli was examined in order to determine whether negative priming would persist across an intervening trial. Second, the number of distractors was manipulated in order to determine whether negative priming is affected by distractor load.

The effect of stimulus repetition on positive and negative identity priming was examined by contrasting familiar performance (i.e., repeated stimuli) with novel performance (i.e., stimuli seen only twice in the experiment-once in the prime display, and once in the probe display). The subjects in Experiments 1 and 2 were presented with novel stimuli, those in Experiments 3 and 4 were presented with repeated stimuli, and those in Experiment 5 were presented with both novel and familiar stimuli in a mixed design. Thus, Experiments $1-5$ constitute the first systematic investigation of the effects of stimulus repetition on negative identity priming.

The effect of an intervening trial on positive and negative identity priming was examined in Experiments 1-4 by contrasting performance when a target on Trial $n$ was an item from the previous trial $(n-1)$ with performance when the target was an item from the penultimate trial (n-2). Some researchers (DeSchepper \& Treisman, 1991; Neill, Valdes, \& Terry, 1992; Tipper, Weaver, Cameron, Brehaut, \& Bastedo, 1991; Treisman, 1992) have suggested that negative priming persists over several inter- 
vening trials, whereas others (Neill et al., 1990; Neill \& Westberry, 1987) have found that negative-priming effects are maximal if the prime-probe interval is shorter than $2 \mathrm{sec}$, and decrease thereafter. The present research examined the extent to which negative priming is modulated by an intervening trial, and determined whether this effect was influenced by stimulus repetition.

The effect of distractor load on positive and negative identity priming was examined by contrasting performance when each trial consisted of a single target paired with a single distractor (Experiments 1 and 3) with performance when each trial consisted of a single target paired with four identical distractors (Experiments 2 and 4). Previous research examining the effect of distractor load on negative priming suggests that negative priming increases with the number of homogeneous distractors (e.g., Humphrey, Kramer, \& Strayer, 1994). In the present research, we increased the number of identical distractors in Experiments 2 and 4 to maximize the likelihood of obtaining negative priming in the IR condition. We did this in order to examine the extent to which negative priming is modulated by the number of identical distractors, and to determine the extent to which stimulus repetition may influence this effect.

\section{EXPERIMENT 1}

Previous research has not examined whether negative priming will be observed in a completely novel IR condition. Most experiments reporting negative priming have used a limited stimulus ensemble. Thus, it is possible that negative priming is dependent on stimulus repetition. Experiment 1 examined positive and negative identity priming effects using novel stimuli. Subjects were presented with a word-naming task consisting of one target word and one distractor word. All words in the experiment were presented either once (filler trials) or twice (prime and probe trials). If negative priming is not dependent on stimulus repetition, negative-priming effects should be obtained under these circumstances.

\footnotetext{
Method

Subjects. Thirty-five University of Utah undergraduates (13 male, 22 female) participated for research credit in an introductory psychology course. They ranged in age from 18 to 27 , with a mean age of 21. All subjects reported normal or corrected-to-normal vision and were native speakers of English.

Stimuli. The stimuli consisted of 1,600 four-to-seven-letter words from the Kučera and Francis (1967) word norms. Word frequencies ranged between 18 and 32 per million. The stimuli were presented above and below a fixation point in the center of the display, and were presented on a black background. Each word was presented either only once (filler trials) or twice (prime and probe trials). The subjects were seated $40 \mathrm{~cm}$ from the display. Each word subtended a visual angle of $0.5^{\circ}$ vertically and $1.7^{\circ}-2.6^{\circ}$ horizontally. The entire array subtended a visual angle of $1.2^{\circ}$ vertically and $1.7^{\circ}-2.6^{\circ}$ horizontally.

Apparatus. The experiment was performed on an IBM-compatible computer with a Viewsonic 6 superVGA monitor. The subjects' responses were measured by a voice-activated response device.
}

Procedure. Each subject participated in a l-h session consisting of 1,000 trials. Each trial consisted of a target word printed in red and a distractor word printed in white. Both the target and the distractor were of equal letter length. The target word on each trial was randomly presented above or below the fixation point, and the distractor was presented in the other location. The target and distractor words remained on the computer screen for $150 \mathrm{msec}$. The subjects' task was to name out loud, as quickly and as accurately as possible, the word printed in red. The latency of the response was measured (to a precision of $1 \mathrm{msec}$ ) with a voice-activated relay, and the accuracy of the response was recorded by the experimenter. The response-stimulus interval (RSI) between response on one trial and the onset of the next display was $500 \mathrm{msec}$. At the end of each block of 100 trials, there was a pause in the experiment to allow subjects to take a brief rest. At the end of the experiment, the subjects were presented with a detailed questionnaire that assessed their awareness of repeated words. Subjects were classified as aware if they indicated any overt awareness of the IR condition.

Awareness was assessed because some researchers (e.g., Driver \& Baylis, 1993) have suggested that this factor may influence negative priming. However, Neill and Valdes (1992) found no difference between subjects who were informed prior to the experiment about the IR condition and subjects who were unaware of the IR condition. We assessed awareness in our subjects to make sure that this factor did not influence our results. There were no significant differences between aware and unaware subjects in any of the experiments reported in this article (all $p \mathrm{~s}>.10$ ).

Design. There were $200 \mathrm{AR}$ and $200 \mathrm{IR}$ prime-probe couplets in the experiment. Each couplet consisted of a prime trial followed by a probe trial. In AR couplets, the target word in the probe trial was the same as the target word in the prime trial. In IR couplets, the target word in the probe trial was the same as the distractor word in the prime trial. All probe-trial distractors were novel words. The AR and IR prime-probe couplets were presented in an unpredictable order.

An additional manipulation in the experiment examined lag effects. In Lag 0 couplets, the probe target was either the target or distractor from the previous trial $(n-1)$. In Lag 1 couplets, the probe target was either the target or the distractor from the penultimate trial $(n-2)$. Lag 0 and Lag 1 prime-probe couplets were equally likely, were presented in an unpredictable order, and were counterbalanced across priming conditions. Thus, the experiment included $100 \mathrm{AR}$ and $100 \mathrm{IR}$ prime-probe couplets with a lag of 0 , and $100 \mathrm{AR}$ and $100 \mathrm{IR}$ prime-probe couplets with a lag of 1 . Targets and distractors for the 200 remaining filler trials were filled with randomly selected novel words.

\section{Results}

The data were analyzed using a $2 \times 2$ (priming condition $\times$ lag) repeated measures analysis of variance (ANOVA). Priming condition refers to AR and IR conditions. Lag refers to Lag 0 and Lag 1 . A significance level of $p<.05$ was adopted for all inferential tests. All planned comparisons were conducted using the Bonferronni $t$ test corrected for familywise error.

Reaction time. The mean of subjects' median RTs for each of the experimental conditions is presented in Table 1. Median RT was greater for IR conditions than for AR conditions $\left[F(1,34)=102.24, M S_{e}=39,850, p<\right.$ $.01]$. The priming condition $\times$ lag interaction $[F(1,34)=$ $\left.6.13, M S_{\mathrm{e}}=1,086, p<.05\right]$ revealed that $\mathrm{RT}$ increased from Lag 0 to Lag 1 for the AR trials, whereas RT for the IR priming conditions did not differ with lag. A planned comparison revealed that RT for filler trials did not differ from that for prime trials. 
Error rate. The error rate (ER) for each of the experimental conditions is presented in Table 1. Overall, ER averaged $3 \%$. The ER was greater for IR conditions than for AR conditions $\left[F(1,34)=38.06, M S_{\mathrm{e}}=112, p<.01\right]$. Neither the main effect of lag nor the interaction (priming condition $\times$ lag) was significant. A planned comparison revealed that $\mathrm{ER}$ for filler trials did not differ from that for prime trials.

Reaction time difference. The effects of AR and IR priming conditions on RT were analyzed by comparing RT for prime targets with RT for probe targets in each of the priming condition $\times$ lag conditions. The RT difference score for each of the conditions is presented in Figure $1 \mathrm{~A}$. A difference score of zero reflects no priming, a positive difference reflects positive priming (i.e., a decrease in RT from prime to probe), and a negative difference reflects negative priming (i.e., an increase in RT from prime to probe). The RT difference was greater for AR conditions than for IR conditions $[F(1,34)=102.43$, $\left.M S_{\mathrm{e}}=39,766, p<.01\right]$. The priming condition $\times$ lag interaction $\left[F(1,34)=6.17, M S_{\mathrm{e}}=1,094, p<.05\right]$ revealed that positive priming for AR trials decreased from Lag 0 to Lag 1, whereas negative priming for IR trials did not differ with lag. Planned comparisons revealed significant positive priming for both $\mathrm{AR}$ conditions, and no negative priming for IR conditions.

Error rate difference. The ER difference score, presented in Figure 1B, was analyzed using the same ANOVA as was used on the RT difference score. A difference score of zero reflects no priming, a positive difference reflects positive priming (i.e., a decrease in ER from prime to probe), and a negative difference reflects negative priming (i.e., an increase in ER from prime to probe). The ER difference score was greater for AR conditions than for IR conditions $\left[F(1,34)=37.99, M S_{\mathrm{e}}=112, p<.01\right]$. Neither the main effect of lag nor the interaction (prim- ing condition $\times$ lag) was significant. Planned comparisons revealed significant positive priming for both $A R$ conditions and no negative priming for IR conditions.

\section{Discussion}

Experiment 1 produced significant positive priming in the AR conditions, whereas negative priming was not obtained in the IR conditions. The lack of negative priming in Experiment 1 challenges the notion that negative priming occurs regardless of experimental stimuli. Specifically, the results suggest that negative priming is not engaged in novel IR conditions. One interpretation of these results is that negative priming is dependent on stimulus repetition. However, before this conclusion can be drawn, it is necessary to replicate these results using a procedure that should enhance the possibility of obtaining negative priming in novel IR conditions.

\section{EXPERIMENT 2}

Experiment 2 was conducted to examine whether negative priming with novel stimuli would be obtained using displays consisting of a target paired with four identical distractors. Humphrey et al. (1994) have recently reported that negative-priming effects are increased as the number of homogeneous distractors increases. The purpose of Experiment 2 was to maximize the likelihood of obtaining negative priming in the novel IR condition by increasing the distractor load. If the results of Experiment 1 are replicated, this will provide support for the hypothesis that negative priming depends on stimulus repetition and is not engaged in novel IR conditions. However, if negative priming is obtained with four identical distractors, it suggests that the selection difficulty in Experiment 1 may not have been sufficient to produce negative priming.

Table 1

Means and Standard Deviations of Median Reaction Time (in Milliseconds) and Percent Error Rate as a Function of Priming Condition and Lag in Experiments $1-4$

\begin{tabular}{|c|c|c|c|c|c|c|c|c|c|c|c|c|}
\hline & & & & & \multicolumn{8}{|c|}{ Priming Condition } \\
\hline & & & & & \multicolumn{4}{|c|}{ Attended Repetition } & \multicolumn{4}{|c|}{ Ignored Repetition } \\
\hline & \multicolumn{2}{|c|}{ Filler } & \multicolumn{2}{|c|}{ Prime } & \multicolumn{2}{|c|}{ Lag 0} & \multicolumn{2}{|c|}{ Lag 1} & \multicolumn{2}{|c|}{ Lag 0} & \multicolumn{2}{|c|}{ Lag 1} \\
\hline & $M$ & $S D$ & $M$ & $S D$ & $M$ & $S D$ & $M$ & $S D$ & $M$ & $S D$ & $M$ & $S D$ \\
\hline \multicolumn{13}{|c|}{ Experiment 1} \\
\hline Median reaction time & 612 & 66 & 614 & 65 & 572 & 58 & 583 & 60 & 611 & 65 & 612 & 65 \\
\hline Error rate & 4.4 & 3.0 & 4.3 & 2.5 & 1.9 & 1.8 & 1.9 & 2.1 & 4.2 & 3.1 & 3.2 & 2.3 \\
\hline \multicolumn{13}{|c|}{ Experiment 2} \\
\hline Median reaction time & 632 & 83 & 633 & 85 & 604 & 82 & 609 & 80 & 629 & 79 & 625 & 85 \\
\hline Error rate & 6.5 & 4.8 & 7.2 & 5.5 & 3.7 & 3.9 & 3.3 & 2.9 & 5.4 & 4.1 & 6.2 & 4.9 \\
\hline \multicolumn{13}{|c|}{ Experiment 3} \\
\hline Median reaction time & 624 & 69 & 547 & 57 & 546 & 52 & 548 & 58 & 562 & 59 & 565 & 63 \\
\hline Error rate & 9.1 & 4.9 & 1.0 & 1.0 & 1.1 & 1.3 & 0.9 & 1.6 & 2.3 & 3.3 & 2.2 & 3.6 \\
\hline \multicolumn{13}{|c|}{ Experiment 4} \\
\hline Median reaction time & 630 & 67 & 553 & 59 & 550 & 60 & 552 & 57 & 565 & 55 & 566 & 55 \\
\hline Error rate & 8.3 & 5.4 & 1.2 & 1.1 & 0.8 & 1.2 & 0.6 & 0.7 & 2.4 & 2.9 & 2.2 & 2.6 \\
\hline
\end{tabular}




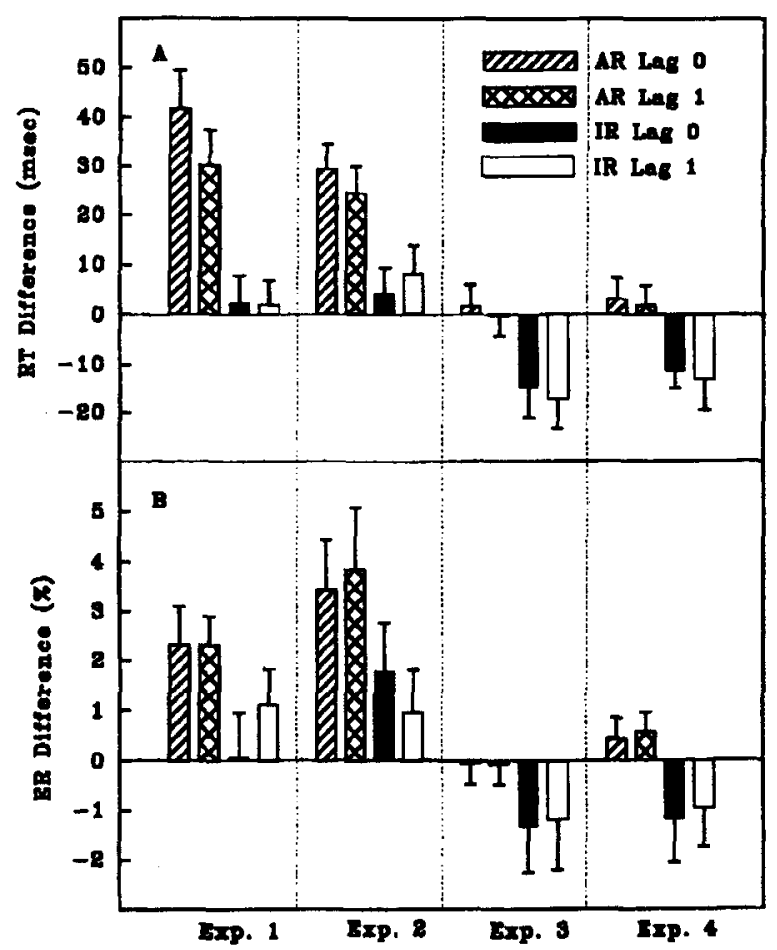

Figure 1. Means of median reaction time (RT, in milliseconds) (panel $A$ ) and percent error rate (ER; panel B), as a function of lag and priming condition (attended repetition [AR] vs. ignored repetition (IR]), in Experiments 1-4. A difference score of zero reflects no priming, a positive difference reflects positive priming, and a negative difference reflects negative priming. Error bars reflect $95 \%$ confidence intervals.

\section{Method}

Subjects. Forty subjects ( 14 male, 26 female) from the same pool of subjects as in Experiment 1 participated. None of these subjects had been a participant in Experiment 1. The subjects were between the ages of 18 and 42, with a mean age of 23 (the age of 4 subjects was not assessed). All subjects reported normal or corrected-to-normal vision and were native speakers of English.

Stimuli and Apparatus. The stimuli were the same as those used in Experiment 1, with the following exception: Four identical distractors were presented in a column of five words in the center of the display; hence, the visual angle of the displays was $3.1^{\circ}$ vertically and $1.7^{\circ}-2.6^{\circ}$ horizontally. The apparatus was the same as that used in Experiment 1.

Procedure and Design. The procedure was the same as that of Experiment 1, with the following exception: Each trial consisted of a target paired with four identical distractors. The four distractor words were always identical and were never the same as the target word. The target word on each trial was randomly presented in one of the three middle locations. The design was the same as that used in Experiment 1.

\section{Results}

Reaction time. The mean of subjects' median RTs for each of the experimental conditions is presented in Table 1. Median RT was greater for IR conditions than for AR conditions $\left[F(1,39)=79.39, M S_{\mathrm{e}}=17,036, p<.01\right]$. Neither the main effect of lag nor the interaction (priming condition $\times$ lag) was significant. A planned com- parison revealed that RT for filler trials did not differ from that for prime trials.

Error rate. The ER for each of the experimental conditions is presented in Table 1. Overall, ER averaged 5\%. The ER was greater for IR conditions than for AR conditions $\left[F(1,39)=28.89, M S_{\mathrm{e}}=200, p<.01\right]$. Neither the main effect of lag nor the interaction (priming condition $\times$ lag) was significant. A planned comparison revealed that $E R$ for filler trials did not differ from that for prime trials.

Reaction time difference. The RT difference score for each of the conditions is presented in Figure 1A. The RT difference score was greater for AR conditions than for IR conditions $\left[F(1,39)=80.44, M S_{\mathrm{e}}=17,087, p<.01\right]$. Neither the main effect of lag nor the interaction (priming condition $\times$ lag) was significant. Planned comparisons revealed significant positive priming for AR conditions and no negative priming for IR conditions.

Error rate difference. The ER difference score for each of the conditions is presented in Figure 1B. It was greater for IR conditions than for AR conditions $[F(1,39)=$ $\left.28.92, M S_{\mathrm{e}}=200, p<.01\right]$. Neither the main effect of lag nor the interaction (priming condition $\times$ lag) was significant. Planned comparisons revealed significant positive priming for both AR conditions and no negative priming for IR conditions.

\section{Discussion}

In Experiment 2, positive priming was obtained in the AR conditions, whereas negative-priming effects were not obtained in the IR conditions. These results demonstrate that the lack of negative priming in Experiment 1 was not due to the number of distractors in each display. Furthermore, Experiment 2 replicates and extends the results of Experiment 1, suggesting that a novel IR condition is not sufficient for a negative-priming mechanism to be engaged.

Together, Experiments 1 and 2 suggest that negative priming is modulated by stimulus repetition. However, before this conclusion can be drawn, it is necessary to determine whether negative priming will be obtained with repeated stimuli using this word-naming task.

\section{EXPERIMENT 3}

The purpose of Experiment 3 was to examine whether the lack of negative priming in Experiments 1 and 2 was due to the use of novel stimuli. The effect of stimulus repetition on positive and negative identity priming was examined by employing a limited stimulus ensemble with the design of Experiment 1 . If negative priming depends on stimulus repetition, the use of a limited stimulus ensemble should be sufficient for negative priming to emerge.

\section{Method}

Subjects. Forty subjects ( 14 male, 26 female) from the same pool of subjects as in Experiments 1 and 2 participated. None of these subjects had been a participant in Experiments 1 or 2. The 
subjects ranged in age from 18 to 35 , with a mean age of 22 . All reported normal or corrected-to-normal vision and were native speakers of English.

Stimuli. The stimuli were the same as those used in Experiment I, with the following exception: The stimuli for the $A R$ and IR prime-probe couplets consisted of 16 words selected randomly without replacement from the same pool of words used in Experiments 1 and 2 . These words were repeated in a random order as targets and distractors for each of the four (priming condition $\times$ lag) conditions. The stimuli for the 200 filler trials were novel words selected randomly without replacement from the same pool of words as that used in Experiments 1 and 2. Each subject saw a different set of repeated and novel words. The apparatus, procedure, and design were the same as those used in Experiment 1 .

\section{Results}

Reaction time. The mean of subjects' median RTs for each of the experimental conditions is presented in Table 1 . Median RT was greater for IR conditions than for AR conditions $\left[F(1,39)=31.13, M S_{\mathrm{e}}=11,072, p<.01\right]$. Neither the main effect of lag nor the interaction (priming condition $\times$ lag) was significant. A planned comparison revealed that RT for filler trials was longer than RT for prime trials.

Error rate. The ER for each of the experimental conditions is presented in Table 1. Overall, ER averaged 2\%. The ER was greater for IR conditions than for AR conditions $\left[F(1,39)=6.54, M S_{\mathrm{e}}=66, p<.01\right]$. Neither the main effect of lag nor the interaction (priming condition $\times$ lag) was significant. A planned comparison revealed that ER for filler trials was greater than ER for prime trials.

Reaction time difference. The RT difference score for each of the conditions is plotted in Figure 1A. The RT difference was greater for IR conditions than for AR conditions $\left[F(1,39)=30.78, M S_{\mathrm{e}}=11,022, p<.05\right]$. Neither the main effect of lag nor the interaction (priming condition $\times$ lag) was significant. Planned comparisons revealed significant negative priming for both IR conditions and no positive priming for AR conditions.

Error rate difference. The ER difference score for each of the conditions is presented in Figure 1B. It was greater for IR conditions than for AR conditions $[F(1,39)=$ $\left.6.53, M S_{\mathrm{e}}=65.8, p<.05\right]$. Neither the main effect of lag nor the interaction (priming condition $\times$ lag) was significant. Planned comparisons revealed significant negative priming for both IR conditions and no positive priming for AR conditions.

\section{Discussion}

The pattern of results in Experiments 1 and 3 reveals a tradeoff in terms of priming effects. The novel conditions of Experiment 1 produced positive priming on AR trials but not negative priming on IR trials, whereas the familiar conditions of Experiment 3 produced negative priming on IR trials but not positive priming on AR trials. The absence of positive priming in Experiment 3 may be attributed to the fact that the repeated stimuli, having been seen many times, were at such high levels of activation that the difference between prime and probe trials in terms of an item's activation level was minimal. In- spection of Table 1 reveals that the RT for prime trials (repeated words) was significantly shorter than the RT for filler trials (novel words), supporting this hypothesis.

The absence of positive priming in Experiment 3, coupled with the presence of negative priming, suggests that a negative-priming mechanism is only engaged when distractors are highly activated. Note that the results are not driven by a speed-accuracy tradeoff; AR conditions in Experiment 1 were faster and more accurate than the neutral condition, whereas IR conditions in Experiment 3 were slower and less accurate than the neutral condition.

Together, the results of Experiments 1 and 3 suggest that negative priming is modulated by stimulus repetition. Our tentative conclusion is that a situation in which distractors are highly activated appears to be necessary for a negative-priming mechanism to be engaged. However, before this conclusion can be drawn, it is important to replicate the pattern of results obtained in Experiment 3.

\section{EXPERIMENT 4}

Experiment 4 was conducted to determine whether the same positive- and negative-priming effects would be obtained with repeated stimuli and displays consisting of a single target and four distractors. Following the logic of Experiment 2, increasing the number of distractors should increase the magnitude of negative priming (e.g., Humphreys et al., 1994). Thus, Experiment 4 employed a limited stimulus ensemble using the design of Experiment 2. A replication of the pattern of results from Experiment 3 would provide strong evidence that negative priming depends on the activation levels of distracting stimuli.

\section{Method}

Subjects. Forty subjects (15 male, 25 female) from the same pool of subjects as those in Experiments 1-3 participated. None of these subjects had been a participant in Experiments 1-3. The subjects ranged in age from 18 to 41 , with a mean age of 20 . All reported normal or corrected-to-normal vision and were native speakers of English.

Stimuli. The stimuli were the same as those used in Experiment 3, with the following exception: Four distractors were presented in each display; hence, the visual angle of the displays was $3.1^{\circ}$ vertically and $1.7^{\circ}-2.6^{\circ}$ horizontally. The apparatus, procedure, and design were the same as those used in Experiment 2.

\section{Results}

Reaction time. The mean of subjects' median RTs for each of the experimental conditions is presented in Table 1. Median RT was greater for IR conditions than for AR conditions $\left[F(1,39)=19.64, M S_{\mathrm{e}}=8,599, p<\right.$ $.01]$. Neither the main effect of lag nor the interaction (priming condition $\times$ lag) was significant. A planned comparison revealed that RT for filler trials was longer than RT for prime trials.

Error rate. The ER for each of the experimental conditions is presented in Table 1. Overall, ER averaged $2 \%$. The ER was greater for IR conditions than for AR conditions $\left[F(1,39)=13.8, M S_{\mathrm{e}}=99, p<.01\right]$. Neither the 
main effect of lag nor the interaction (priming condition $\times$ lag) was significant. A planned comparison revealed that the ER for filler trials was greater than the ER for prime trials.

Reaction time difference. The RT difference score for each of the conditions is plotted in Figure 1A. It was greater for IR conditions than for AR conditions $[F(1,39)$ $\left.=19.61, M S_{\mathrm{e}}=8,606, p<.01\right]$. Neither the effect of lag nor the interaction (priming condition $\times$ lag) was significant. Planned comparisons revealed significant negative priming for both IR conditions and no positive priming for AR conditions.

Error rate difference. The ER difference score for each of the conditions is plotted in Figure 1B. It was greater for IR conditions than for AR conditions $[F(1,39)=$ $\left.13.88, M S_{\mathrm{e}}=99.35, p<.01\right]$. Neither the effect of lag nor the interaction (priming condition $\times$ lag) was significant. Planned comparisons revealed significant negative priming for both IR conditions and significant positive priming for Lag 1 of the AR condition.

\section{Discussion}

Experiment 4 produced significant negative priming for IR conditions. With the exception of an effect of ER at Lag 1, no positive priming effects were obtained in the AR conditions. This pattern of results replicates and extends the pattern of results of Experiments 1 and 3, providing strong support for the conclusion that negative priming depends on a situation in which distractors are highly activated.

\section{Additional Analysis}

A between-experiment analysis was performed to determine whether there were significant effects of stimulus repetition and number of distractors. A $2 \times 2 \times 2 \times$ 2 (repetition $\times$ number of distractors $\times$ priming condition $\times$ lag) split-plot ANOVA was performed on the RT difference and ER difference data from Experiments 1-4. So as not to be redundant with previous analyses, we focus our discussion only on the main effects and interactions involving repetition and number of distractors. First, the analysis revealed a main effect of repetition $\left[F(1,151)=162.11, M S_{\mathrm{e}}=551, \dot{p}<.01\right]$, indicating that the RT difference score was greater for novel stimuli than for repeated stimuli. More importantly, there was a repetition $\times$ priming condition interaction $[F(1,151)=$ $\left.14.81, M S_{\mathrm{e}}=348, p<.01\right]$. The means for novel stimuli were 31.3 and $4.2 \mathrm{msec}$ for AR and IR conditions, respectively. The means for repeated stimuli were 1.5 and $-14.1 \mathrm{msec}$ for AR and IR conditions, respectively. Planned comparisons revealed significant positive priming for the novel AR and IR conditions, and significant negative priming only for the repeated IR condition. In addition, there was a main effect of repetition on the ER difference score $\left[F(1,151)=73.91, M S_{\mathrm{e}}=13, p<.01\right]$. The means for the novel stimuli were $3.01 \%$ and $0.99 \%$ error for AR and IR conditions, respectively. The means for the repeated stimuli were $0.25 \%$ and $-1.18 \%$ error for AR and IR conditions, respectively. Post hoc analy- ses revealed that the difference in ER between the prime and probe trials was greater for novel stimuli. There were no systematic effects of number of distractors.

Together, Experiments 1-4 reveal a tradeoff in terms of priming. Experiments 1 and 2 (nonrepeated stimuli) resulted in positive priming for the AR conditions, but did not produce negative priming in the IR conditions. In fact, novel IR conditions resulted in significant positive priming, albeit quite small. In Experiments 3 and 4 (repeated stimuli), negative priming was produced for the IR conditions, but positive priming was not produced in the AR conditions. The novel conditions produced positive but not negative priming, whereas the familiar conditions produced negative but not positive priming.

\section{EXPERIMENT 5}

The results of Experiments 1-4 demonstrate that negative priming is modulated by stimulus repetition. Specifically, a situation in which distractors are highly activated is necessary for the engagement of a negative-priming mechanism. The presence of negative priming in Experiments 3 and 4, coupled with the absence of negative priming in Experiments 1 and 2, demonstrates that negativepriming effects emerge only when highly activated items are presented in tandem and compete for a response. However, this conclusion may be premature, because there is a possibility that these effects may be driven by strategic differences between experimental conditions. When experimental conditions are manipulated between subjects or in blocked designs, it is possible that any differences between conditions are due to differential strategies. This problem can be avoided by using a within-subjects design in which novel and repeated stimuli are presented in an unpredictable order (e.g., Sperling \& Dosher, 1986). If the pattern of results obtained in Experiments 1-4 is replicated in a mixed design, strategic differences between conditions cannot be said to have produced the effects. Alternatively, if the pattern of effects in Experiment 5 differs from that of Experiments 1-4, it would suggest that these differences are due to differential strategies.

The purpose of Experiment 5 was to perform a withinsubjects replication of Experiments $1-4$. Because neither the manipulation of number of distractors nor the manipulation of lag produced any systematic effects in Experiments $1-4$, these factors were not manipulated in $\mathrm{Ex}$ periment 5.

\section{Method}

Subjects. Thirty-five subjects ( 14 male, 21 female) from the same pool of subjects as in Experiments 1-4 participated. None of these subjects had been a participant in Experiments 1-4. The subjects ranged in age from 18 to 38 , with a mean age of 22 . All reported normal or corrected-to-normal vision and were native speakers of English.

Stimuli. The stimuli were the same as those used in the preceding experiments, with the following exception: The stimuli consisted of 616 words selected randomly without replacement from the same pool of words as that used in Experiments 1-4. Sixteen words formed the stimulus ensemble for the repeated AR and IR 
Table 2

Means and Standard Deviations of Median Reaction Time

(in Milliseconds) and Percent Error Rate as a Function of Stimulus

Repetition (Repeated vs. Novel) and Priming Condition in Experiment 5

\begin{tabular}{|c|c|c|c|c|c|c|c|c|c|c|c|c|c|c|c|c|}
\hline & \multicolumn{16}{|c|}{ Priming Condition } \\
\hline & \multicolumn{8}{|c|}{ Attended Repetition } & \multicolumn{8}{|c|}{ Ignored Repetition } \\
\hline & \multicolumn{4}{|c|}{ Repeated } & \multicolumn{4}{|c|}{ Novel } & \multicolumn{4}{|c|}{ Repeated } & \multicolumn{4}{|c|}{ Novel } \\
\hline & \multicolumn{2}{|c|}{ Prime } & \multicolumn{2}{|c|}{ Probe } & \multicolumn{2}{|c|}{ Prime } & \multicolumn{2}{|c|}{ Probe } & \multicolumn{2}{|c|}{ Prime } & \multicolumn{2}{|c|}{ Probe } & \multicolumn{2}{|c|}{ Prime } & \multicolumn{2}{|c|}{ Probe } \\
\hline & $M$ & $S D$ & $M$ & $S D$ & $M$ & $S D$ & $M$ & $S D$ & $M$ & $S D$ & $M$ & $S D$ & $M$ & $S D$ & $M$ & $S D$ \\
\hline RT & 564 & 58 & 546 & 57 & 612 & 58 & 555 & 57 & 564 & 58 & 575 & 59 & 612 & 58 & 603 & 60 \\
\hline ER & 2.1 & 1.9 & 1.7 & 2.1 & 7.0 & 4.2 & 3.0 & 3.3 & 2.1 & 1.9 & 2.3 & 2.3 & 7.0 & 4.2 & 5.8 & 4.9 \\
\hline
\end{tabular}

prime-probe couplets. The words were selected as targets and distractors in a random order. Each word was presented an average of 56 times during the experiment. The stimulus ensemble for the novel AR and IR prime-probe couplets was formed by 600 additional words. These words were repeated only twice during the experiment- once in a prime display, and once in a probe display. The apparatus and procedure were the same as those used in Experiment 3.

Design. The design was the same as that of Experiment 3, with the following exceptions: First, because there were no systematic effects of lag in Experiments 1-4, Experiment 5 included only Lag 0 . Second, the 200 filler trials were replaced with 50 repeated $\mathrm{AR}$ and 50 repeated IR prime-probe couplets. This was done to maintain a high level of activation for the repeated stimuli. Thus, the experiment included $150 \mathrm{AR}$ and $150 \mathrm{IR}$ repeated prime-probe couplets, and $100 \mathrm{AR}$ and $100 \mathrm{IR}$ novel prime-probe couplets. The presentation order of novel and familiar couplets and priming condition was completely randomized.

\section{Results}

The data were analyzed with a $2 \times 2$ (repetition $\times$ priming condition) repeated measures ANOVA. Repetition refers to repeated and novel couplets; priming condition refers to AR and IR conditions.

Reaction time. Median RT for each of the experimental conditions is presented in Table 2. Median RT was greater for IR than for AR probe trials $[F(1,34)=127.44$, $\left.M S_{\mathrm{e}}=51,532, p<.01\right]$, and was greater for novel probe trials than for repeated probe trials $[F(1,34)=33.63$, $\left.M S_{\mathrm{e}}=12,635, p<.01\right]$. The repetition $\times$ priming condition interaction $\left[F(1,34)=19.88, M S_{\mathrm{e}}=3,361, p<.01\right]$ revealed a greater difference between novel and repeated probe trials for IR conditions than for AR conditions.

Error rate. The ER for each of the experimental conditions is presented in Table 2. Overall, ER averaged 3\%. The ER was greater for IR than for AR probe trials $[F(1,34)$ $\left.=20.95, M S_{\mathrm{e}}=97, p<.01\right]$, and was greater for novel than for repeated probe trials $\left[F(1,34)=32.23, M S_{\mathrm{e}}=\right.$ $211, p<.01]$. The repetition $\times$ priming condition interaction $\left[F(1,34)=10.75, M S_{\mathrm{e}}=41, p<.01\right]$ revealed a greater difference between novel and repeated probe trials for IR conditions than for AR conditions.

Reaction time difference. The RT difference score for each of the conditions is plotted in Figure 2A. It was greater for AR probe trials than for IR probe trials $\left[F(1,34)=127.58, M S_{\mathrm{e}}=51,609, p<.01\right]$, and was greater for novel than for repeated probe trials $[F(1,34)=$ $\left.153.70, M S_{\mathrm{e}}=29,842, p<.01\right]$. The repetition $\times$ priming condition interaction $\left[F(1,34)=19.65, M S_{\mathrm{e}}=3,341\right.$, $p<.01]$ revealed a greater difference between novel and repeated probe trials for AR conditions than for IR conditions. Planned comparisons revealed significant positive priming in both repeated and novel AR conditions, as well as in the novel IR condition. Negative priming was observed only in the repeated IR condition. Thus, these data replicate and extend the findings obtained in Experiments 1-4.

Error rate difference. The ER difference score for each of the conditions is presented in Figure 2B. It was greater for AR probe trials than for IR probe trials $[F(1,34)=$ $\left.20.92, M S_{\mathrm{e}}=97, p<.01\right]$, and was greater for novel than for repeated probe trials $\left[F(1,34)=42.92, M S_{\mathrm{e}}=215\right.$, $p<.01]$. The repetition $\times$ priming condition interaction

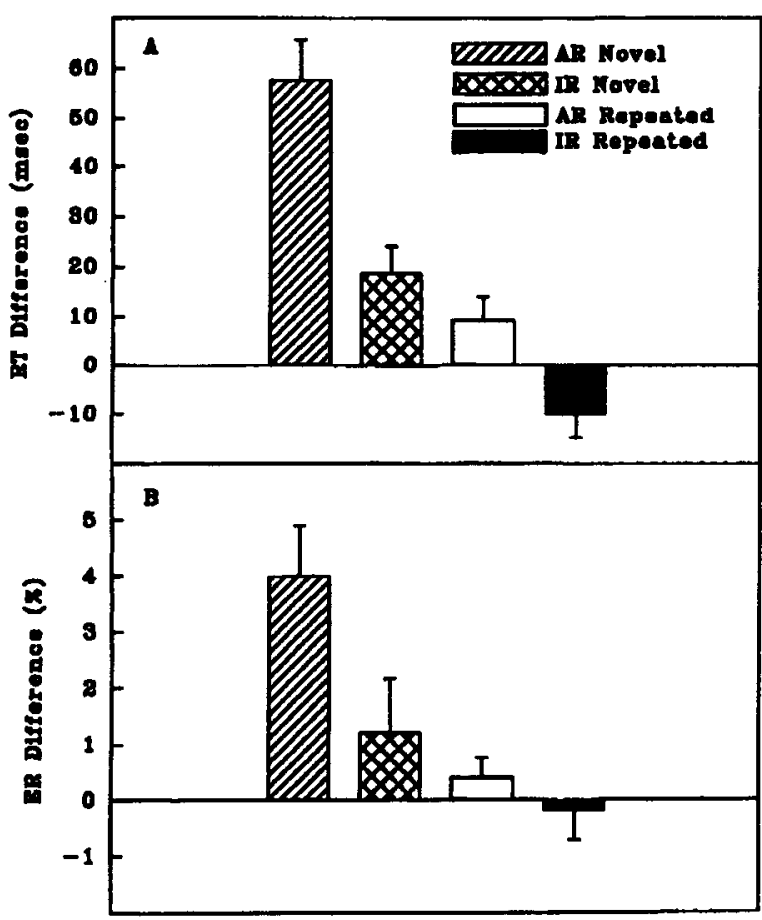

Figure 2. Means of median reaction time (RT, in milliseconds) (panel $A$ ) and percent error rate (ER; panel B), as a function of priming condition (attended repetition [AR] vs. ignored repetition [IR]), in Experiment 5. A difference score of zero reflects no priming, a positive difference reflects positive priming, and a negative difference reflects negative priming. Error bars reflect $95 \%$ confidence intervals. 
$\left[F(1,34)=10.75, M S_{\mathrm{e}}=41, p<.01\right]$ revealed a greater ER difference between novel and repeated probe trials for AR conditions than for IR conditions. Planned comparisons revealed significant positive priming in both repeated and novel AR conditions, as well as in the novel IR condition.

\section{Discussion}

In Experiment 5, repeated primes were responded to faster and more accurately than novel primes, suggesting that repeated words were more highly activated than novel words. Positive priming in the AR condition was greater for novel than for repeated stimuli. Negative priming was observed only in the repeated IR condition. Moreover, positive priming was obtained in the novel IR condition, demonstrating that low activation levels can lead to positive priming regardless of whether an item is attended or ignored in the prime display. Thus, the data replicate and extend the pattern of data obtained in Experiments $1-4$. This finding is important because it suggests that the differences between novel and repeated conditions are not due to differential strategies. Moreover, the distractor stimuli must have been processed to the level of identification in order for negative priming to be stimulus specific. This suggests that the mechanism(s) underlying negative priming occur relatively late in the information-processing sequence. In summary, the results of Experiment 5 demonstrate that negative priming is dependent on stimulus repetition, and that it is stimulus specific.

It is important to make one additional observation with respect to Experiment 5: The magnitude of negative priming in the repeated IR condition of Experiment 5 was smaller than that obtained in Experiments 3 and 4. The reduction in negative priming can be attributed to the fact that repeated stimuli were presented about half as often in Experiment 5 as they were in Experiments 3 and 4 . These results suggest that there is a monotonic relationship between stimulus repetition and the magnitude of negative priming.

\section{GENERAL DISCUSSION}

In Experiments 1 and 2, in which novel stimuli were used, positive priming was found in the AR conditions, but not negative priming in the IR conditions. In Experiments 3 and 4 , in which repeated stimuli were used, negative priming was found in the IR conditions, but not positive priming in the AR conditions. The novel conditions produced positive but not negative priming, whereas the familiar conditions produced negative but not positive priming. Moreover, the cross-experimental analysis (i.e., across Experiments 1-4) revealed that the novel IR condition resulted in positive priming. This finding was replicated in Experiment 5.

The results demonstrate that negative priming is modulated by stimulus repetition. Specifically, a situation in which distractors are highly activated is necessary for the engagement of a negative-priming mechanism. The pres- ence of negative priming in Experiments 3 and 4, coupled with the absence of negative priming in Experiments 1 and 2, demonstrates that negative-priming effects emerge only when highly activated items are presented in tandem and compete for a response. This finding was replicated within subjects in Experiment 5, demonstrating that the effect of repetition on negative priming cannot be explained by differential strategies.

Experiment 5 also demonstrated that negative priming is stimulus specific. Negative priming was obtained only on probe trials in which the target was a previously ignored distractor that had been repeatedly presented during the experiment. The results also suggest that negative priming occurs relatively late during processing, because some type of stimulus identification must have taken place in order to determine whether or not to engage the negative-priming mechanism(s).

The present results appear to be at odds with those reported by DeSchepper and Treisman (1991; see also Treisman, 1992), in which a single presentation of a novel shape resulted in negative priming for that shape. DeSchepper and Treisman interpreted their results as supporting a negative-priming mechanism driven by the maintenance of specific internal representations of distracting stimuli. However, negative priming for novel stimuli in DeSchepper and Treisman's studies may be an artifact of the way in which the subjects categorized the highly similar shapes. DeSchepper and Treisman also used spatially overlapping stimuli in their experiments. It is possible that overlapping stimuli increased the selection difficulty, thereby augmenting negative-priming effects. A final possibility is that the effects of stimulus repetition may be more pronounced for verbal than for spatial stimuli. Further research will be required to resolve this issue.

The effects of stimulus repetition on positive and negative priming may be explained in terms of the levels of activation of stimuli used in the experiment. This activationmodel interpretation proposes that negative-priming effects emerge when two highly activated items are presented in tandem and compete for a response. With repeated stimuli, item-activation levels are high, and negative priming will be obtained because selection difficulty is high. That is, when the distractor is highly activated, it is more likely to interfere with responding to the target. It is in such circumstances that the mechanisms underlying negative priming are activated.

Positive priming, measured as the difference in processing between prime and probe, will not be obtained with repeated stimuli because activation levels are close to asymptote. Thus, differences in activation levels between the prime and probe will be minimal; however, there should be large differences between a repeated probe and the first presentation of that stimulus (e.g., a filler trial in Experiments 3 and 4), consistent with the repetitionpriming literature (e.g., Logan, 1990).

With novel stimuli, item activation levels are low, and negative priming will not be obtained because selection difficulty is low; that is, novel distractors are less likely to 
interfere with responding to the target. In these circumstances, the mechanisms underlying negative priming are not engaged. Positive priming should be obtained with novel stimuli because there is a relatively large difference in the activation levels on the prime and probe trials. In a mixed design including both novel and repeated stimuli, negative priming will be obtained only for repeated stimuli and positive priming will be obtained only for novel stimuli. Although Experiments 1-4 obtained no differences between negative priming at Lags 0 and 1 , the activation model predicts that increasing the number of intervening trials so as to decrease item activation should decrease negative priming. Further research will be required to test this prediction.

Finally, the analysis of Experiments 1-4 revealed that there was no systematic effect of distractor load. Previous research examining the effect of distractor load on negative priming has produced mixed results. Some research suggests that negative priming increases as the number of homogeneous distractors increases (Humphrey et al., 1994), whereas other research suggests that negative priming decreases as the number of heterogeneous distractors increases (Neumann \& DeSchepper, 1992). Unfortunately, there appears to be no clear-cut pattern emerging with respect to the effect of distractor load on negative priming.

\section{Implications for Theories of Negative Priming}

There are three major theories concerning the mechanism underlying negative priming. First, the deactivation model (Neill, 1979) attributes negative-priming effects to a reduction in the activation levels of the mental representations of distracting stimuli. This model proposes that positive and negative priming reflect similar but inverse mechanisms: Positive priming reflects the activation of mental representations above a base level of activation, whereas negative priming reflects the inhibition of mental representations below a base level of activation.

The data are problematic for the deactivation model, because novel IR conditions resulted in significant positive priming, suggesting that the activation of these items was not inhibited below baseline levels. Furthermore, relative to novel filler trials, all repeated stimuli were processed faster and with fewer errors, providing further evidence that activation levels were not suppressed below baseline. Thus, these data are inconsistent with the predictions of the deactivation model.

Second, the episodic retrieval model (Neill \& Valdes, 1992) attributes negative priming to the retrieval of an incompatible memory trace on the probe trial. The processes underlying the episodic retrieval model are similar to those underlying Logan's (1988) instance theory of automaticity. According to the episodic retrieval model, each time a stimulus is presented, a new memory trace is formed. One attribute included in the memory trace concerns whether the stimulus was a target or distractor. Performance on subsequent trials is de- termined by a race between an algorithmic process of identifying the target and the retrieval of prior instances. If an incompatible instance (i.e., an instance in which the target stimulus was previously a distractor) is retrieved, it will interfere with processing, resulting in negative priming.

At first glance, the effects of stimulus repetition on negative priming may seem to be consistent with the episodic retrieval model. However, the episodic retrieval model has difficulty accounting for several aspects of the data. First, because the only instance that is available on a novel probe trial is an incompatible one (i.e., an instance from the prime trial), the episodic retrieval model predicts that negative priming should be greater on novel trials than on repeated trials (in which both compatible and incompatible traces would be present). Furthermore, it is not clear that the episodic retrieval model would produce negative priming on repeated trials if a stimulus had been seen as a target and as a distractor equally often. Under these conditions, the episodic retrieval model predicts positive priming if a compatible trace was the first instance to be retrieved and negative priming if an incompatible trace was the first instance to be retrieved. If the probability of retrieving an incompatible trace is equal to the probability of retrieving a compatible trace, there should be no negative priming on repeated trials. Thus, these data are inconsistent with the predictions of the episodic retrieval model.

Third, the blocking model (Tipper \& Cranston, 1985) proposes that the mental representations of distracting items are blocked from access to response mechanisms. This model attributes negative-priming effects to the maintenance of a "selection state" in which the information-processing system must distinguish and select between targets and distractors in order to determine the response. Thus, negative priming may not occur on probe trials in which there is no selection requirement (Neill, Terry, \& Valdes, 1994; but see Moore, 1994). Note that in the blocking model, no deactivation is required; the mental representations of distractors may remain activated while being blocked from access to response mechanisms.

The blocking model is consistent with our activationmodel interpretation of the data. Specifically, negative priming is stimulus specific, and emerges when there is competition between targets and distractors for response. However, the blocking model must be amended so that only highly activated distractors are blocked from access to response mechanisms. This follows, because novel IR conditions resulted in significant positive priming. If all distractors were blocked from access to response mechanisms, negative priming should not be affected by stimulus repetition.

In summary, Experiments 1-5 demonstrate that negative priming is stimulus specific and will be obtained only in a situation in which stimuli are at high levels of activation. These results appear to be most consistent with a modified response-blocking model of negative priming. 


\section{REFERENCES}

Beech, A., Baylis, G. C., Smithson, P., \& Claridge, G. (1989). Individual differences in schizotypy as reflected in cognitive measures of inhibition. British Journal of Clinical Psychology, 28, 117-129.

BEECH, A., \& ClaRIDGE, G. (1987). Individual differences in negative priming: Relations with schizotypal personality traits. British Journal of Psychology, 78, 349-356.

BroAdBEnT, D. E. (1958). Perception and communication. London: Pergamon Press.

DeSchePPER, B., \& Treisman, A. (1991, November). Novel visual shapes in negative priming. Paper presented at the 32 nd annual meeting of the Psychonomic Society, San Francisco.

DrIVER, J., \& BAYLIS, G. C. (1993). Cross-modal negative priming and interference in selective attention. Bulletin of the Psychonomic Society, 31, 45-48.

Hasher, L., Kane, M., Stoltzfus, E. R., Zacks, R. T., \& Connelly, $S$. L. (in press). Inhibitory attentional mechanisms and aging. Psychology \& Aging.

Humphrey, D. G., Kramer, A. F., \& Strayer, D. L. (1994, April). Negative priming and aging: The effects of pacing and the number of distractors. Paper presented at the Sixth Cognitive Aging Conference, Atlanta.

KuČERA, H., \& FRANCIS, W. N. (1967). Computational analysis of present-day American English. Providence, RI: Brown University Press.

LoGAN, G. D. (1988). Towards an instance theory of automatization. Psychological Review, 85, 492-527.

LogAN, G. D. (1990). Repetition priming and automaticity: Common underlying mechanisms? Cognitive Psychology, 22, 1-35.

Moore, C. M. (1994). Negative priming depends on probe-trial conflict: Where has all the inhibition gone? Perception \& Psychophysics, 56, 133-147.

NEILL, W. T. (1977). Inhibitory and facilitatory processes in selective attention. Journal of Experimental Psychology: Human Perception \& Performance, $3,444-450$.

NeILL, W. T. (1979). Switching attention within and between categories: Evidence for intracategory inhibition. Memory \& Cognition, 7, 283-290.

NeILl, W. T., LisSNER, L. S., \& BECK, J. L. (1990). Negative priming in same-different matching: Further evidence for a central locus of inhibition. Perception \& Psychophysics, 48, 398-400.

Neill, W. T., Terry, K. M., \& Valdes, L. A. (1994). Negative priming without probe selection. Psychonomic Bulletin \& Review, 1, 119. 121.

NeILL, W. T., \& VALDES, L. A. (1992). Persistence of negative priming: Steady state or decay? Journal of Experimental Psychology: Learning, Memory, \& Cognition, 18, 565-576.

NeIll, W. T., Valdes, L. A., \& Terry, K M. (1992, November). Negative priming in target localization. Paper presented to the meeting of the Psychonomic Society, St. Louis.

NeIL., W. T., \& WESTBERRY, R. L. (1987). Selective attention and the suppression of cognitive noise. Journal of Experimental Psychology: Learning, Memory, \& Cognition, 13, 327-334

NeumanN, E., \& DeSchepper, B. G. (1992). An inhibition based fan effect: Evidence for an active suppression mechanism in selective attention. Canadian Journal of Psychology, 46, 11-50.

Neumann, E., McCloskey, M. S., \& Felio, A. C. (1993). Primed lexical decision tasks: Cross-cultural positive priming disappears, negative priming doesn't. Manuscript submitted for publication.

Rosch, E. (1975). Cognitive representations of semantic categories. Journal of Experimental Psychology: General, 104, 192-233.

SPERLING, G., \& Dosher, B. (1986). Strategy and optimization in human information processing. In K. R. Boff, L. Kaufman, \& J. P. Thomas (Eds.), Handbook of perception and human performance (Vol. 1, pp. 2-1 to 2-65). New York: Wiley.

TIPPER, S. P. (1985). The negative priming effect: Inhibitory priming by ignored objects. Quarterly Journal of Experimental Psychology, 37, $571-590$.

TIPPER, S. P., \& BAYLIS, G. C. (1987). Individual differences in selective attention: The relation of priming and interference to cognitive failure. Personality \& Individual Differences, 8, 667-675.

TiPPER, S. P., Brehaut, J. C., \& Driver, J. (1990). Selection of moving and static objects for the control of spatially-directed action. Journal of Experimental Psychology: Human Perception \& Performance, 16, 492-504.

TIPPER, S. P., \& CRANSTON, M. (1985). Selective attention and priming: Inhibitory and facilitatory effects of ignored primes. Quarterly Journal of Experimental Psychology, 37A, 591-611.

TIPPER, S. P., \& DRIVER, J. (1988). Negative priming between pictures and words in a selective attention task: Evidence for semantic processing of ignored stimuli. Memory \& Cognition, 16, 64-70.

Tipper, S. P., MacQueEN, G. M., \& Brehaut, J. C. (1988). Negative priming between response modalities: Evidence for the central locus of inhibition in selective attention. Perception \& Psychophysics, 43, 45-52.

Tipper, S. P., Weaver, B., Cameron, S., Brehaut, J. C., \& Bastedo, J. (1991). Inhibitory mechanisms of attention in identification tasks: Time-course and disruption. Journal of Experimental Psychology: Learning, Memory, \& Cognition, 17, 681-692.

Treisman, A. M. (1964). Monitoring and storage of irrelevant messages in selective attention. Journal of Verbal Learning \& Verbal Behavior, 3, 449-459.

Treisman, A. M. (1992). Perceiving and re-perceiving objects. American Psychologist, 47, 862-875.

VAN DER HEIJDEN, A. H. C. (1981). Short term visual information processing. London: Routledge \& Kegan Paul.

YeE, PENNY L. (1991). Semantic inhibition of ignored words during a figure classification task. Quarterly Journal of Experimental Psychology, 43A, 127-153.

(Manuscript received May 5, 1994; revision accepted for publication December 15, 1994.) 